\title{
About Exact Solution of Some Non Linear Partial Integro-differential Equations
}

\author{
Francis Bassono $^{1}$, Rasmané Yaro ${ }^{2}$, Joseph Bonazebi Yindoula ${ }^{3}$, Gires Dimitri Nkaya ${ }^{3}$, \\ Gabriel Bissanga ${ }^{3}$ \\ ${ }^{1}$ Faculty of Sciences, Joseph KI-ZERBO University, Ouagadougou, Burkina Faso \\ ${ }^{2}$ Faculty of Sciences, Dedougou University, Dedougou, Burkina Faso \\ ${ }^{3}$ Faculty of Sciences and Technology, Marien NGOUABI University, Brazzaville, Congo
}

\section{Email address:}

bonayindoula@yahoo.fr (J. B. Yindoula)

${ }^{*}$ Corresponding author

\section{To cite this article:}

Francis Bassono, Rasmané Yaro, Joseph Bonazebi Yindoula, Gires Dimitri Nkaya, Gabriel Bissanga. Inferences on the Weibull Exponentiated Exponential Distribution and Applications. Applied and Computational Mathematics. Vol. 10, No. 1, 2021, pp. 19-29.

doi: 10.11648/j.acm.20211001.13

Received: January 15, 2021; Accepted: February 24, 2021; Published: March 30, 2021

\begin{abstract}
Data on solving of nonlinear integro-differential equations using Laplace-SBA method are scarce. The objective of this paper is to determine exact solution of nonlinear 2 dimensionnal Voltera-Fredholm differential equation by this method. First, SBA method and Laplace SBA method are described. Second, three nonlinear Voolterra-Fredholm integro-differential equations are solved using each method. Application of each method give an exact solution. However, application of Laplace-SBA method permits for solve integro-differential equation compared with SBA method. This proves that this last method can be fruitfully applied in the resolution of integro-differential equations.
\end{abstract}

Keywords: Partial Integro-differential Equation, Volterra-Fredholm Equation, SBA Method, Laplace SBA Method

\section{Introduction}

For 50 years, we have observed the renewal of an intimate link between numerical analysis and physics (theoretical and applied). The two scientific fields become more and more complementary and the methods developed in one are fruitfully applied in the other. Indeed, the partial integrodifferential equations (linear and nonlinear) are involved in the modeling of several phenomena and mechanisms observed in engineering sciences (mechanics of structures and fluid dynamics, process engineering, etc), in physics (propagation of waves, field theory, etc) and even in biology $[1,2]$. It is in this context that their resolution has interested mathematicians, especially the specialists in numerical analysis. To this proposal, several researchers have devoted their work to the search of exact or approximate solutions of nonlinear integro-differential equations using various methods, such as triangular function [3], reduced differential transformation method [4], Tau method [5].

In this paper, we solve some nonlinear integro-differential equations by the Laplace-Somé Blaise Abbo (SBA) method initiated by African mathematicians, particularly those of Burkina Faso [6], and Congo-Brazzaville [7, 8].

\section{Description of SBA Method and Laplace-SBA Method}

\subsection{SBA Method}

About SBA method, we can see $[6,7]$

\subsection{Laplace-SBA Method: [9, 10]}

Consider the following equation:

$$
A u=f
$$

$A: H \longrightarrow H$ is a linear or a nonlinear operator $\mathrm{H}$ is a real Hilbert space, $f \in H$ and $u$ is the unknown. 
We suppose that we can decomposite the operator $A$ in the following form:

$$
A=L+R+N
$$

Where $L+R$, is the linear part and $N$, the non-linear part, $\mathrm{L}$ is supposed invertible in the sens of Adomoian with $\mathrm{L}^{-1}$ as inverse.

Then, equation (1) gives

$$
L u+R u+N u=g
$$

Applying the Laplace transform $\mathcal{L}$ to $(3)$, we obtain :

$$
\mathcal{L}(L u)+\mathcal{L}(R u)+\mathcal{L}(N u)=\mathcal{L}(g)
$$

Let's Consider the case where : $L()=.\frac{\partial^{2}}{\partial t^{2}}($.

Then

$\mathcal{L}\left[\frac{\partial^{2}(u(x, t))}{\partial t^{2}}\right]=s^{2} \mathcal{L}(u(x, t))-s u(x, 0)-\frac{\partial u(x, 0)}{\partial t}$

Thus, equation (4) becomes:

$$
\begin{aligned}
& s^{2} \mathcal{L}(u(x, t))-s u(x, 0)-\frac{\partial u(x, 0)}{\partial t}+\mathcal{L}(R u)+\mathcal{L}(N u)=\mathcal{L}(g) \\
\Leftrightarrow & \\
& \mathcal{L}(u(x, t))=-\frac{1}{s^{2}} \mathcal{L}(R u(x, t))-\frac{1}{s^{2}} \mathcal{L}(N u(x, t))+\frac{1}{s} u(x, 0)+\frac{1}{s^{2}} \frac{\partial u(x, 0)}{\partial t}+\frac{1}{s^{2}} \mathcal{L}(g)
\end{aligned}
$$

According to the successive approximations method from (7), we have:

$$
\mathcal{L}\left(u^{k}(x, t)\right)=-\frac{1}{s^{2}} \mathcal{L}\left(R u^{k}(x, t)\right)-\frac{1}{s^{2}} \mathcal{L}\left(N u^{k-1}(x, t)\right)+\frac{1}{s} u(x, 0)+\frac{1}{s^{2}} \frac{\partial u(x, 0)}{\partial t}+\frac{1}{s^{2}} \mathcal{L}(g), k \geq 1
$$

We look for the solution of (1) in a serie expansion form:

$$
u^{k}(x, t)=\sum_{n=0}^{+\infty} u_{n}^{k}(x, t)
$$

Taking (9) in to (8), we obtain:

$$
\begin{aligned}
\sum_{n=0}^{+\infty}\left(\mathcal{L}\left(u_{n}^{k}(x, t)\right)\right) & =-\sum_{n=0}^{+\infty}\left(\frac{1}{s^{2}} \mathcal{L}\left(R u_{n}^{k}(x, t)\right)\right)-\frac{1}{s^{2}} \mathcal{L}\left(N u^{k-1}(x, t)\right) \\
& +\frac{1}{s} u(x, 0)+\frac{1}{s^{2}} \frac{\partial u(x, 0)}{\partial t}+\frac{1}{s^{2}} \mathcal{L}(g), k \geq 1
\end{aligned}
$$

According to the SBA method, we suppose that the solution of $(1)$ is $u(x, t)=\lim _{k \rightarrow+\infty} u^{k}(x, t)$ and for every $k \geq 1$, we get $u_{n}^{k}(x, t)$ for $n \geq 0$ the following SBA algorithm:

We deduce the following SBA algorithm:

$$
\left\{\begin{array}{l}
\mathcal{L}\left(u_{0}^{k}(x, t)\right)=\frac{1}{s} u(x, 0)+\frac{1}{s^{2}} \frac{\partial u(x, 0)}{\partial t}+\frac{1}{s^{2}} \mathcal{L}(g)-\frac{1}{s^{2}} \mathcal{L}\left(N u^{k-1}(x, t)\right) \\
\mathcal{L}\left(u_{n+1}^{k}(x, t)\right)=-\left(\frac{1}{s^{2}} \mathcal{L}\left(R u_{n}^{k}(x, t)\right)\right), n \geq 0
\end{array}\right.
$$

Applying the inverse transform $\mathcal{L}^{-1}$ Laplace to (11), we have:

$$
\left\{\begin{array}{l}
\left(u_{0}^{k}(x, t)\right)=\mathcal{L}^{-1}\left(\frac{1}{s} u(x, 0)\right)+\mathcal{L}^{-1}\left(\frac{1}{s^{2}} \frac{\partial u(x, 0)}{\partial t}\right)+\mathcal{L}^{-1}\left(\frac{1}{s^{2}} \mathcal{L}(g)\right)-\mathcal{L}^{-1}\left(\frac{1}{s^{2}} \mathcal{L}\left(N u^{k-1}(x, t)\right)\right) \\
u_{n+1}^{k}(x, t)=-\mathcal{L}^{-1}\left(\frac{1}{s^{2}} \mathcal{L}\left(R u_{n}^{k}(x, t)\right)\right), \forall n \geq 0
\end{array}\right.
$$

The SBA principle needs that, for $k=1$, we must choose $u^{0}(x, t)$ like $N\left(u^{0}\right)=0$ and for $k>1$, we must have $N\left(u^{k-1}\right)=0$.

The solution at each step is given by:

$$
u=\lim _{k \rightarrow+\infty} u^{k}=\lim _{k \rightarrow+\infty} \sum_{n \geq 0} u_{n}^{k}
$$




\section{Numerical Application}

We apply SBA method and Laplace-SBA method to solve three nonlinear integro-differential equations.

\subsection{Example 1: $[3,13]$}

In this example, we consider the following a two-dimensional mixed Volterra-Fredholm integro-differential equation:

$$
\left\{\begin{array}{l}
u_{x x}+u_{x t}(x, t)+\int_{0}^{1} \int_{0}^{1}\left(x t+y z^{2}\right) u(y, z) d y d z+u^{3}(x, t)+ \\
\int_{0}^{t} \int_{0}^{x}(x+t+y+z) u^{2}(y, z) d y d z=g(x, t),(x, t) \in[0,1]^{2} \\
u(0, t)=0 \\
u_{x}(0, t)=e^{t}
\end{array}\right.
$$

where

$$
\begin{aligned}
g(x, t) & =-\frac{1}{2} x t-\frac{2}{3}+\frac{1}{2} t e^{x}+\frac{1}{3} e-\frac{7}{24} x^{4}-\frac{1}{6} x^{3} t+\frac{1}{12} x^{3} \\
& +\frac{7}{24} x^{4} e^{2 t}+\frac{1}{3} t x^{3} e^{2 t}-\frac{1}{12} x^{3} e^{2 t}+e^{t}+x^{3} e^{3 t}
\end{aligned}
$$

Resolution by the SBA method

Consider the equation

$$
u_{x x}=g(x, t)-\left(u_{x t}(x, t)+\int_{0}^{1} \int_{0}^{1}\left(x t+y z^{2}\right) u(y, z) d y d z\right)-\left(u^{3}(x, t)+\int_{0}^{t} \int_{0}^{x}(x+t+y+z) u^{2}(y, z) d y d z\right)
$$

From (16), we obtain:

$$
\begin{aligned}
u(x, t) & \left.=x e^{t}+\int_{0}^{x}\left(\int_{0}^{\lambda} g(s, t) d s\right) d \lambda-\int_{0}^{x}\left(\int_{0}^{\lambda} u_{s t}(s, t) d s\right) d \lambda-\int_{0}^{x}\left(\int_{0}^{\lambda} \int_{0}^{1} \int_{0}^{1}\left(s t+y z^{2}\right) u(y, z) d y d z\right) d s\right) d \lambda \\
& -\int_{0}^{x}\left(\int_{0}^{\lambda}\left(u^{3}(s, t)\right) d s\right) d \lambda-\int_{0}^{x}\left(\int_{0}^{\lambda}\left(\int_{0}^{t} \int_{0}^{s}(s+t+y+z) u^{2}(y, z) d y d z\right) d s\right) d \lambda
\end{aligned}
$$

Thus

$$
\begin{aligned}
u(x, t) & =x e^{t}+\frac{1}{6} x^{2} e-\frac{1}{12} t x^{3}+\frac{1}{2} x^{2} e^{t}-\frac{1}{3} x^{2}+\frac{1}{12} t x^{3} e+N(u) \\
& -\int_{0}^{x}\left(\int_{0}^{\lambda} u_{s t}(s, t) d s\right) d \lambda-\int_{0}^{x}\left(\int_{0}^{\lambda}\left(\int_{0}^{1} \int_{0}^{1}\left(s t+y z^{2}\right) u(y, z) d y d z\right) d s\right) d \lambda
\end{aligned}
$$

where

$$
\left\{\begin{aligned}
N(u(x, t)) & =-\frac{1}{120} t x^{5}-\frac{1}{240} x^{5} e^{2 t}+\frac{1}{20} x^{5} e^{3 t}+\frac{7}{720} x^{6} e^{2 t}+\frac{1}{240} x^{5} \\
& -\frac{7}{720} x^{6}+\frac{1}{60} t x^{5} e^{2 t}+\int_{0}^{x}\left(\int_{0}^{\lambda}\left(u^{3}(s, t)\right) d s\right) d \lambda \\
& -\int_{0}^{x}\left(\int_{0}^{\lambda}\left(\int_{0}^{t} \int_{0}^{s}(s+t+y+z) u^{2}(y, z) d y d z\right) d s\right) d \lambda
\end{aligned}\right.
$$

Applying the successive approximations method to (18), we have:

$$
\left\{\begin{array}{l}
u^{k}(x, t)=x e^{t}+\frac{1}{6} x^{2} e-\frac{1}{12} t x^{3}+\frac{1}{2} x^{2} e^{t}-\frac{1}{3} x^{2}+\frac{1}{12} t x^{3} e+N\left(u^{k-1}\right) \\
-\int_{0}^{x}\left(\int_{0}^{\lambda} u_{s t}^{k}(s, t) d s\right) d \lambda-\int_{0}^{x}\left(\int_{0}^{\lambda}\left(\int_{0}^{1} \int_{0}^{1}\left(s t+y z^{2}\right) u^{k}(y, z) d y d z\right) d s\right) d \lambda,
\end{array}\right.
$$


According to the SBA method, we find the solution of problem (1), through the followind modified Adomian algorithm:

$$
\left\{\begin{aligned}
u_{0}^{k}(x, t) & =x e^{t}+N\left(u^{k-1}\right), k \geq 1 \\
u_{1}^{k}(x, t) & =\frac{1}{6} x^{2} e-\frac{1}{12} t x^{3}+\frac{1}{2} x^{2} e^{t}-\frac{1}{3} x^{2}+\frac{1}{12} t x^{3} e-\int_{0}^{x}\left(\int_{0}^{\lambda}\left(u_{0}\right)_{s t}^{k}(s, t) d s\right) d \lambda \\
& -\int_{0}^{x}\left(\int_{0}^{\lambda}\left(\int_{0}^{1} \int_{0}^{1}\left(s t+y z^{2}\right) u_{0}^{k}(y, z) d y d z\right) d s\right) d \lambda \\
u_{n+1}^{k}(x, t) & =-\int_{0}^{x}\left(\int_{0}^{\lambda}\left(u_{n}\right)_{s t}^{k}(s, t) d s\right) d \lambda-\int_{0}^{x}\left(\int_{0}^{\lambda}\left(\int_{0}^{1} \int_{0}^{1}\left(s t+y z^{2}\right) u_{n}^{k}(y, z) d y d z\right) d s\right) d \lambda, n \geq 0
\end{aligned}\right.
$$

For $k=1$, we have the following SBA algorithm:

$$
\left\{\begin{aligned}
u_{0}^{1}(x, t)= & x e^{t}+N\left(u^{0}\right) \\
u_{1}^{1}(x, t)= & \frac{1}{6} x^{2} e-\frac{1}{12} t x^{3}+\frac{1}{2} x^{2} e^{t}-\frac{1}{3} x^{2}+\frac{1}{12} t x^{3} e-\int_{0}^{x}\left(\int_{0}^{\lambda}\left(u_{0}\right)_{s t}^{1}(s, t) d s\right) d \lambda- \\
& \int_{0}^{x}\left(\int_{0}^{\lambda}\left(\int_{0}^{1} \int_{0}^{1}\left(s t+y z^{2}\right) u_{0}^{1}(y, z) d y d z\right) d s\right) d \lambda \\
u_{n+1}^{1}(x, t)= & -\int_{0}^{x}\left(\int_{0}^{\lambda}\left(u_{n}\right)_{s t}^{1}(s, t) d s\right) d \lambda-\int_{0}^{x}\left(\int_{0}^{\lambda}\left(\int_{0}^{1} \int_{0}^{1}\left(s t+y z^{2}\right) u_{n}^{1}(y, z) d y d z\right) d s\right) d \lambda
\end{aligned}\right.
$$

From (23), we have get:

$$
\left\{\begin{array}{l}
u_{0}^{1}(x, t)=x e^{t} \\
u_{1}^{1}(x, t)=\left(\frac{1}{6} x^{2} e+\frac{1}{2} x^{2} e^{t}-\frac{1}{3} x^{2}-\frac{1}{12} t x^{3}+\frac{1}{12} t x^{3} e\right)+\left(-\frac{1}{6} x^{2} e-\frac{1}{2} x^{2} e^{t}+\frac{1}{3} x^{2}+\frac{1}{12} x^{3} t-\frac{1}{12} x^{3} t e\right)=0 \\
u_{n}^{1}(x, t)=0
\end{array}\right.
$$

So the solution in step 1 is:

$$
u^{1}(x, t)=\sum_{n=0}^{\infty} u_{n}^{1}(x, t)=u_{0}^{1}(x, t)=x e^{t} \operatorname{car} \forall n \geq 1 u_{n}^{1}(x, t)=0
$$

Thus, we get the solution in step 1:

$$
u^{1}(x, t)=x e^{t}
$$

For $k=2$, we have following SBA algorithm:

$$
\left\{\begin{aligned}
u_{0}^{2}(x, t) & =x e^{t}+N\left(u^{1}\right), k \geq 1 \\
u_{1}^{2}(x, t) & =\frac{1}{6} x^{2} e-\frac{1}{12} t x^{3}+\frac{1}{2} x^{2} e^{t}-\frac{1}{3} x^{2}+\frac{1}{12} t x^{3} e-\int_{0}^{x}\left(\int_{0}^{\lambda}\left(u_{0}\right)_{s t}^{2}(s, t) d s\right) d \lambda \\
& -\int_{0}^{x}\left(\int_{0}^{\lambda}\left(\int_{0}^{1} \int_{0}^{1}\left(s t+y z^{2}\right) u_{0}^{2}(y, z) d y d z\right) d s\right) d \lambda \\
u_{n+1}^{2}(x, t) & =-\int_{0}^{x}\left(\int_{0}^{\lambda}\left(u_{n}\right)_{s t}^{2}(s, t) d s\right) d \lambda-\int_{0}^{x}\left(\int_{0}^{\lambda}\left(\int_{0}^{1} \int_{0}^{1}\left(s t+y z^{2}\right) u_{n}^{2}(y, z) d y d z\right) d s\right) d \lambda, n \geq 0
\end{aligned}\right.
$$


First, let's calculate $N\left(u^{1}\right)$.

$$
\left\{\begin{aligned}
N\left(u^{1}\right) & =-\frac{1}{120} t x^{5}-\frac{1}{240} x^{5} e^{2 t}+\frac{1}{20} x^{5} e^{3 t}+\frac{7}{720} x^{6} e^{2 t}+\frac{1}{240} x^{5} \\
& -\frac{7}{720} x^{6}+\frac{1}{60} t x^{5} e^{2 t}-\int_{0}^{x}\left(\int_{0}^{\lambda} s^{3} e^{3 t} d s\right) d \lambda \\
& -\int_{0}^{x}\left(\int_{0}^{\lambda}\left(\int_{0}^{t} \int_{0}^{s}(s+t+y+z) y^{2} e^{2 z} d y d z\right) d s\right) d \lambda \\
& =-\frac{1}{120} t x^{5}-\frac{1}{240} x^{5} e^{2 t}+\frac{1}{20} x^{5} e^{3 t}+\frac{7}{720} x^{6} e^{2 t}+\frac{1}{240} x^{5} \\
& -\frac{7}{720} x^{6}+\frac{1}{60} t x^{5} e^{2 t}-\frac{1}{20} x^{5} e^{3 t}+\frac{6}{720} x^{5} t+\frac{7}{720} x^{5} x \\
& +\frac{3}{720} x^{5} e^{2 t}-\frac{12}{720} x^{5} t e^{2 t}-\frac{7}{720} x^{5} x e^{2 t}-\frac{3}{720} x^{5} \\
& =0
\end{aligned}\right.
$$

Therefore, the modified SBA algorithm (26) allows to write:

$$
\left\{\begin{aligned}
u_{0}^{2}(x, t) & =x e^{t}+N\left(u^{1}\right) \\
u_{1}^{2}(x, t) & =\frac{1}{6} x^{2} e-\frac{1}{12} t x^{3}+\frac{1}{2} x^{2} e^{t}-\frac{1}{3} x^{2}+\frac{1}{12} t x^{3} e-\int_{0}^{x}\left(\int_{0}^{\lambda}\left(u_{0}\right)_{s t}^{2}(s, t) d s\right) d \lambda \\
- & \int_{0}^{x}\left(\int_{0}^{\lambda}\left(\int_{0}^{1} \int_{0}^{1}\left(s t+y z^{2}\right) u_{0}^{2}(y, z) d y d z\right) d s\right) d \lambda \\
u_{n+1}^{1}(x, t) & =-\int_{0}^{x}\left(\int_{0}^{\lambda}\left(u_{n}\right)_{s t}^{2}(s, t) d s\right) d \lambda-\int_{0}^{x}\left(\int_{0}^{\lambda}\left(\int_{0}^{1} \int_{0}^{1}\left(s t+y z^{2}\right) u_{n}^{2}(y, z) d y d z\right) d s\right) d \lambda
\end{aligned}\right.
$$

$\Leftrightarrow$

$$
\left\{\begin{array}{l}
u_{0}^{2}(x, t)=x e^{t} \\
u_{1}^{2}(x, t)=\left(\frac{1}{6} x^{2} e+\frac{1}{2} x^{2} e^{t}-\frac{1}{3} x^{2}-\frac{1}{12} t x^{3}+\frac{1}{12} t x^{3} e\right)+\left(-\frac{1}{6} x^{2} e-\frac{1}{2} x^{2} e^{t}+\frac{1}{3} x^{2}+\frac{1}{12} x^{3} t-\frac{1}{12} x^{3} t e\right)=0 \\
u_{n}^{2}(x, t)=0
\end{array}\right.
$$

The exact solution in step $k=2$ is

$$
u^{2}(x, t)=x e^{t}
$$

The solution is deduced by recurrence at each step:

$$
u^{2}(x, t)=u^{3}(x, t)=\cdots=u^{k}(x, t)=x e^{t}
$$

and the exact solution of problem 1 is given by:

$$
u(x, t)=\lim _{k \rightarrow+\infty} u^{k}(x, t)=x e^{t}
$$

\subsection{Example 2: $[4,11,12]$}

Consider the following integro-differential equation:

$$
\left\{\begin{array}{l}
u_{t t}+t^{2} u(x, t)-\int_{0}^{t} \int_{0}^{x} y e^{3 z} u^{3}(y, z) d y d z=g(x, t),(x, t) \in[0,1]^{2} \\
u(x, 0)=0 \\
u_{t}(x, 0)=e^{x}
\end{array}\right.
$$


where

$$
g(x, t)=\left(t^{3}+t-2\right) e^{x-t}-\frac{1}{36}(3 x-1) t^{4} e^{3 x}-\frac{1}{36} t^{4}
$$

Resolution by the Laplace-SBA method

Consider the equation

$$
u_{t t}+t^{2} u(x, t)-\int_{0}^{t} \int_{0}^{x} y e^{3 z} u^{3}(y, z) d y d z=g(x, t)
$$

Applying the Laplace transform $\mathcal{L}$ to $(35)$, we have:

$$
\mathcal{L}\left(u_{t t}\right)+\mathcal{L}\left(t^{2} u(x, t)\right)-\mathcal{L}\left(\int_{0}^{t} \int_{0}^{x} y e^{3 z} u^{3}(y, z) d y d z\right)=\mathcal{L}(g(x, t))
$$

$\Leftrightarrow$

$$
s^{2} \mathcal{L}(u(x, t))-s u(x, 0)-\frac{\partial u(x, 0)}{\partial t}+\mathcal{L}\left(t^{2} u(x, t)\right)-\mathcal{L}\left(\int_{0}^{t} \int_{0}^{x} y e^{3 z} u^{3}(y, z) d y d z\right)=\mathcal{L}(g(x, t))
$$

so

$$
\mathcal{L}(u(x, t))=\frac{1}{s^{2}} e^{x}-\frac{1}{s^{2}} \mathcal{L}\left(t^{2} u(x, t)\right)+\frac{1}{s^{2}} \mathcal{L}\left(\int_{0}^{t} \int_{0}^{x} y e^{3 z} u^{3}(y, z) d y d z\right)+\frac{1}{s^{2}} \mathcal{L}(g(x, t))
$$

Applying th inverse transform applied to equation (38), we obtain:

$$
u(x, t)=t e^{x}-\mathcal{L}^{-1}\left(\frac{1}{s^{2}} \mathcal{L}\left(t^{2} u(x, t)\right)\right)+\mathcal{L}^{-1}\left(\left(\frac{1}{s^{2}} \mathcal{L}\left(\int_{0}^{t} \int_{0}^{x} y e^{3 z} u^{3}(y, z) d y d z\right)\right)\right)+\mathcal{L}^{-1}\left(\frac{1}{s^{2}} \mathcal{L}(g(x, t))\right)
$$

We remark that:

$$
\left\{\begin{aligned}
\mathcal{L}^{-1}\left(\frac{1}{s^{2}} \mathcal{L}(g(x, t))\right) & =\mathcal{L}^{-1}\left(\frac{1}{s^{2}}\left[\mathcal{L}\left(\left(t^{3}+t-2\right) e^{x-t}-\frac{1}{36}(3 x-1) t^{4} e^{3 x}-\frac{1}{36} t^{4}\right)\right]\right) \\
& =\left(\frac{1}{36} e^{3 x}-\frac{1}{12} x e^{3 x}\right) \frac{1}{30} t^{6}+e^{x}\left(2-2 e^{-t}-2 t\right)+e^{x}\left(t+2 e^{-t}+t e^{-t}-2\right) \\
& +e^{x}\left(6 t+24 e^{-t}+18 t e^{-t}+6 t^{2} e^{-t}+t^{3} e^{-t}-24\right)-\frac{1}{1080} t^{6}
\end{aligned}\right.
$$

Therefore, we get:

$$
\left\{\begin{aligned}
u(x, t) & =t e^{x}+\left(\frac{1}{36} e^{3 x}-\frac{1}{12} x e^{3 x}\right) \frac{1}{30} t^{6}+\left(2 e^{x}-2 e^{x} e^{-t}-2 e^{x} t\right) \\
& +t e^{x}+2 e^{x} e^{-t}+t e^{x} e^{-t}-2 e^{x}+6 e^{x} t+24 e^{x} e^{-t}+18 t e^{x} e^{-t}+6 t^{2} e^{x} e^{-t} \\
& +t^{3} e^{x} e^{-t}-24 e^{x}-\frac{1}{1080} t^{6}-\mathcal{L}^{-1}\left(\frac{1}{s^{2}} \mathcal{L}\left(t^{2} u(x, t)\right)\right) \\
& +\mathcal{L}^{-1}\left(\left(\frac{1}{s^{2}} \mathcal{L}\left(\int_{0}^{t} \int_{0}^{x} y e^{3 z} u^{3}(y, z) d y d z\right)\right)\right)
\end{aligned}\right.
$$

Thus

$$
\begin{aligned}
u(x, t) & =t e^{x}+24 e^{x} e^{-t}-24 e^{x}+5 t e^{x}+19 t e^{x} e^{-t}+6 t^{2} e^{x} e^{-t}+t^{3} e^{x} e^{-t} \\
& -\mathcal{L}^{-1}\left(\frac{1}{s^{2}} \mathcal{L}\left(t^{2} u(x, t)\right)\right)+N(u(x, t))
\end{aligned}
$$

where

$$
N(u(x, t))=\mathcal{L}^{-1}\left(\frac{1}{s^{2}} \mathcal{L}\left(\int_{0}^{t} \int_{0}^{x} y e^{3 z} u^{3}(y, z) d y d z\right)\right)-\frac{1}{1080} t^{6}\left(3 x e^{3 x}-e^{3 x}+1\right)
$$


Applying the successive approximation method to (42), we have:

$$
\begin{aligned}
u^{k}(x, t) & =t e^{x}+24 e^{x} e^{-t}-24 e^{x}+5 t e^{x}+19 t e^{x} e^{-t}+6 t^{2} e^{x} e^{-t}+t^{3} e^{x} e^{-t} \\
& -\mathcal{L}^{-1}\left(\frac{1}{s^{2}} \mathcal{L}\left(t^{2} u^{k}(x, t)\right)\right)+N\left(u^{k-1}(x, t)\right), \forall k \geq 1
\end{aligned}
$$

From (44), we have the following modified SBA algorithm:

$$
\left\{\begin{array}{l}
u_{0}^{k}(x, t)=t e^{x} e^{-t}+N\left(u^{k-1}(x, t)\right) \\
u_{1}^{k}(x, t)=24 e^{x} e^{-t}-24 e^{x}+6 t e^{x}+18 t e^{x} e^{-t}+6 t^{2} e^{x} e^{-t}+t^{3} e^{x} e^{-t}-\mathcal{L}^{-1}\left(\frac{1}{s^{2}} \mathcal{L}\left(t^{2} u_{0}^{k}(x, t)\right)\right) \forall k \geq 1 \\
u_{n+1}^{k}(x, t)=-\mathcal{L}^{-1}\left(\frac{1}{s^{2}} \mathcal{L}\left(t^{2} u_{n}^{k}(x, t)\right)\right), \forall n \geq 1
\end{array}\right.
$$

For $k=1$ we have the following SBA algorithm:

$$
\left\{\begin{aligned}
u_{0}^{1}(x, t) & =t e^{x} e^{-t}+N\left(u^{0}(x, t)\right) \\
u_{1}^{1}(x, t) & =24 e^{x} e^{-t}-24 e^{x}+6 t e^{x}+18 t e^{x} e^{-t}+6 t^{2} e^{x} e^{-t}+t^{3} e^{x} e^{-t} \\
& -\mathcal{L}^{-1}\left(\frac{1}{s^{2}} \mathcal{L}\left(t^{2} u_{0}^{1}(x, t)\right)\right) \\
u_{n+1}^{1}(x, t) & =-\mathcal{L}^{-1}\left(\frac{1}{s^{2}} \mathcal{L}\left(t^{2} u_{n}^{1}(x, t)\right)\right), \forall n \geq 1
\end{aligned}\right.
$$

We suppose that one can find $u^{0}$ as $N\left(u^{0}\right)=0$, from (46), we obtain:

$$
\left\{\begin{array}{l}
u_{0}^{1}(x, t)=t e^{x} e^{-t} \\
u_{1}^{1}(x, t)=24 e^{x} e^{-t}-24 e^{x}+6 t e^{x}+18 t e^{x} e^{-t}+6 t^{2} e^{x} e^{-t}+t^{3} e^{x} e^{-t}- \\
\left(6 e^{x} t+24 e^{x} e^{-t}+18 t e^{x} e^{-t}+6 t^{2} e^{x} e^{-t}+t^{3} e^{x} e^{-t}-24 e^{x}\right)=0 \\
u_{n}^{1}(x, t)=0, \forall n \geq 2
\end{array}\right.
$$

From (47), we get:

$$
u^{1}(x, t)=u_{0}^{1}(x, t)=t e^{x-t}
$$

For $k=2$, calculate $N_{1}\left(u^{1}(x, t)\right)$

$$
\left\{\begin{aligned}
N_{1}\left(u^{1}\right) & =\mathcal{L}^{-1}\left(\frac{1}{s^{2}} \mathcal{L}\left(\int_{0}^{t} \int_{0}^{x} y e^{3 z} u^{3}(y, z) d y d z\right)\right)-\frac{1}{1080} t^{6}\left(3 x e^{3 x}-e^{3 x}+1\right) \\
& =\left(3 x e^{3 x}-e^{3 x}+1\right) \mathcal{L}^{-1}\left(\frac{2}{3 s^{7}}\right)-\frac{1}{1080} t^{6}\left(3 x e^{3 x}-e^{3 x}+1\right) \\
& =\left(3 x e^{3 x}-e^{3 x}+1\right) \frac{1}{1080} t^{6}-\frac{1}{1080} t^{6}\left(3 x e^{3 x}-e^{3 x}+1\right) \\
& =0
\end{aligned}\right.
$$

The SBA algorithm gives:

$$
\left\{\begin{array}{l}
u_{0}^{2}(x, t)=t e^{x} e^{-t} \\
u_{1}^{2}(x, t)=24 e^{x} e^{-t}-24 e^{x}+6 t e^{x}+18 t e^{x} e^{-t}+6 t^{2} e^{x} e^{-t}+t^{3} e^{x} e^{-t}-\mathcal{L}^{-1}\left(\frac{1}{s^{2}} \mathcal{L}\left(t^{2} u_{0}^{2}(x, t)\right)\right) \\
u_{n+1}^{2}(x, t)=-\mathcal{L}^{-1}\left(\frac{1}{s^{2}} \mathcal{L}\left(t^{2} u_{n}^{2}(x, t)\right)\right), \forall n \geq 1
\end{array}\right.
$$


From (50), we get:

$$
\left\{\begin{aligned}
u_{0}^{2}(x, t) & =t e^{x} e^{-t} \\
u_{1}^{2}(x, t) & =24 e^{x} e^{-t}-24 e^{x}+6 t e^{x}+18 t e^{x} e^{-t}+6 t^{2} e^{x} e^{-t}+t^{3} e^{x} e^{-t} \\
& -\left(6 e^{x} t+24 e^{x} e^{-t}+18 t e^{x} e^{-t}+6 t^{2} e^{x} e^{-t}+t^{3} e^{x} e^{-t}-24 e^{x}\right)=0 \\
u_{n}^{2}(x, t) & =0, \forall n \geq 2
\end{aligned}\right.
$$

Thus:

$$
u^{2}(x, t)=u_{0}^{2}(x, t)=t e^{x-t}
$$

Using the same procedure we obtain:

$$
u^{2}(x, t)=u^{3}(x, t)=\cdots=u^{k}(x, t)=t e^{x-t}
$$

and the solution of $(33)$ is:

$$
u(x, t)=\lim _{k \rightarrow+\infty} u^{k}(x, t)=t e^{x-t}
$$

\subsection{Example 3: [3]}

Consider the following integro-differential equation:

$$
\left\{\begin{array}{l}
u_{t t}+u(x, t)-\int_{0}^{t} \int_{0}^{x}(y-\cos z) u^{2}(y, z) d y d z+\int_{0}^{1} \int_{0}^{1} u^{3}(y, z) d y d z=g(x, t),(x, t) \in[0,1]^{2} \\
u(x, 0)=0 \\
u_{t}(x, 0)=x
\end{array}\right.
$$

where

$$
g(x, t)=\frac{1}{8} x^{4} \cos t \sin t-\frac{1}{8} x^{4} t-\frac{1}{9} x^{3} \sin t+\frac{1}{9} x^{3} \sin t \cos ^{2}(t)+\frac{1}{6}-\frac{1}{4} \cos (1)+\frac{1}{12} \cos ^{3}(1)
$$

Resolution by the SBA method

Let's denote

$$
\begin{aligned}
& L u=\frac{\partial^{2} u}{\partial t^{2}}, \\
& R u(x, t)=u(x, t) \\
& N(u(x, t))=g(x, t)+\int_{0}^{t} \int_{0}^{x}(y+\cos z) u^{2}(y, z) d y d z-\int_{0}^{1} \int_{0}^{1} u^{3}(y, z) d y d z
\end{aligned}
$$

The equation (55) gives:

$$
L u+R u=N u
$$

From (58), we obtain:

$$
u(x, t)=u(x, 0)+t \frac{\partial u}{\partial t}(x, 0)-\int_{0}^{t} \int_{0}^{s} R u(x, v) d v d s+\int_{0}^{t} \int_{0}^{s} N u(x, v) d v d s
$$

$\Leftrightarrow$

$$
u(x, t)=x t-\int_{0}^{t} \int_{0}^{s} u(x, v) d v d s+\int_{0}^{t} \int_{0}^{s} N u(x, v) d v d s
$$

let's denote

$$
\widetilde{N}(u(x, t))=\int_{0}^{t} \int_{0}^{s} N u(x, v) d v d s
$$

Applying the successive approximations method to (60), we have:

$$
u^{k}(x, t)=x t+\widetilde{N}\left(u^{k-1}(x, t)\right)-\int_{0}^{t} \int_{0}^{s} u^{k}(x, v) d v d s ; k \geq 1
$$


From (62), we get the following SBA algorithm:

$$
\begin{cases}u_{0}^{k}(x, t)= & x t+\tilde{N}\left(u^{k-1}(x, t)\right) ; k \geq 1 \\ u_{n+1}^{k}(x, t)= & -\int_{0}^{t} \int_{0}^{s} u_{n}^{k}(x, v) d v d s ; n \geq 0\end{cases}
$$

For $k=1$, we suppose that one can find $\mathrm{u}^{0}$ as $N\left(u^{0}\right)=0$, we obtain the following SBA algorithm :

$$
\begin{cases}u_{0}^{1}(x, t)= & x t \\ u_{n+1}^{1}(x, t)= & -\int_{0}^{t} \int_{0}^{s} u_{n}^{1}(x, v) d v d s, \forall n \geq 0\end{cases}
$$

From (64), we get :

$$
\left\{\begin{aligned}
u_{0}^{1}(x, t) & =x t \\
u_{1}^{1}(x, t) & =-\frac{1}{6} x t^{3} \\
u_{2}^{1}(x, t) & =\frac{1}{120} x t^{5} \\
\ldots & \\
u_{n}^{1}(x, t) & =\frac{(-1)^{n}}{(2 n+1) !} x t^{2 n+1}
\end{aligned}\right.
$$

From (65), we have :

$$
u^{1}=\sum_{n=0}^{\infty} u_{n}^{1}=x \sum_{n=0}^{\infty} \frac{(-1)^{n}}{(2 n+1) !} t^{2 n+1}=x \sin t
$$

For $k=2$, we have the following algorithm :

$$
\begin{cases}u_{0}^{2}(x, t)= & x t+\tilde{N}\left(u^{1}(x, t)\right) \\ u_{n+1}^{2}(x, t)= & -\int_{0}^{t} \int_{0}^{s} u_{n}^{2}(x, v) d v d s, ; n \geq 0\end{cases}
$$

We calculate:

$$
\tilde{N}\left(u^{1}(x, t)\right)=\int_{0}^{t} \int_{0}^{s} N u^{1}(x, v) d v d s
$$

where

$$
\begin{aligned}
& N(u(x, t))=g(x, t)+\underbrace{\int_{0}^{t} \int_{0}^{x}(y+\cos z)\left[u^{1}(y, z)\right]^{2} d y d z}_{A}-\underbrace{\int_{0}^{1} \int_{0}^{1}\left[u^{1}(y, z)\right]^{3} d y d z}_{B} \\
& \left.A=\int_{0}^{t} \int_{0}^{x}(y+\cos z)\left[u^{1}(y, z)\right]^{2} d y d z=\int_{0}^{t} \int_{0}^{x}(y+\cos z) y^{2} \sin ^{2} z\right) d y d z \\
& =\int_{0}^{t} \sin ^{2} z\left(\int_{0}^{x}\left(y^{3}+y^{2} \cos z\right) d y\right) d z=\int_{0}^{t}\left(\frac{1}{4} x^{4} \sin ^{2} z+\frac{1}{3} x^{3}\left(\sin ^{2} z \cos z\right)\right) d z \\
& =\int_{0}^{t}\left(\frac{1}{8} x^{4}(1-\cos 2 z)-\frac{1}{12} x^{3}(\cos 3 z-\cos z)\right) d z=-\frac{1}{8} x^{4} \cos t \sin t+\frac{1}{8} t x^{4}-\frac{1}{36} x^{3} \sin 3 t+\frac{1}{12} x^{3} \sin t \\
& =-\frac{1}{8} x^{4} \cos t \sin t+\frac{1}{8} t x^{4}-\frac{1}{36} x^{3}\left(4 \cos ^{2} t \sin t-\sin t\right)+\frac{1}{12} x^{3} \sin t \\
& =-\frac{1}{8} x^{4} \sin t \cos t+\frac{1}{8} t x^{4}-\frac{1}{9} x^{3} \sin t \cos ^{2} t+\frac{1}{9} x^{3} \sin t
\end{aligned}
$$


and

$$
\begin{aligned}
B & =\int_{0}^{1} \int_{0}^{1}\left[u^{1}(y, z)\right]^{3} d y d z=\int_{0}^{1} \int_{0}^{1} y^{3} \sin ^{3} z d y d z \\
& =\frac{1}{48} \cos 3-\frac{3}{16} \cos 1+\frac{1}{6} \\
& =\frac{1}{48}\left(4 \cos ^{3} 1-3 \cos 1\right)-\frac{3}{16} \cos 1+\frac{1}{6} \\
& =\frac{1}{12} \cos ^{3} 1-\frac{1}{4} \cos 1+\frac{1}{6}
\end{aligned}
$$

Therefore

$$
\begin{aligned}
N\left(u^{1}(x, t)\right) & =g(x, t)+A-B \\
& =\frac{1}{8} x^{4} \cos t \sin t-\frac{1}{8} x^{4} t-\frac{1}{9} x^{3} \sin t+\frac{1}{9} x^{3} \sin t \cos ^{2}(t)+\frac{1}{6}-\frac{1}{4} \cos (1) \\
& +\frac{1}{12} \cos ^{3}(1)+\left(-\frac{1}{8} x^{4} \sin t \cos t+\frac{1}{8} t x^{4}-\frac{1}{9} x^{3} \sin t \cos ^{2} t+\frac{1}{9} x^{3} \sin t\right) \\
& -\left(\frac{1}{12} \cos ^{3} 1-\frac{1}{4} \cos 1+\frac{1}{6}\right) \\
& =0
\end{aligned}
$$

So

$$
\widetilde{N}\left(u^{1}(x, t)\right)=\int_{0}^{t} \int_{0}^{s} N u^{1}(x, v) d v d s=0
$$

(67) gives :

$$
\begin{cases}u_{0}^{2}(x, t)= & x t \\ u_{n+1}^{2}(x, t)= & -\int_{0}^{t} \int_{0}^{s} u_{n}^{2}(x, v) d v d s, \forall n \geq 0\end{cases}
$$

Thus, we get :

We have :

$$
\left\{\begin{aligned}
u_{0}^{2}(x, t) & =x t \\
u_{1}^{2}(x, t) & =-\frac{1}{6} x t^{3} \\
u_{2}^{2}(x, t) & =\frac{1}{120} x t^{5} \\
\ldots & \\
u_{n}^{2}(x, t) & =\frac{(-1)^{n}}{(2 n+1) !} x t^{2 n+1}
\end{aligned}\right.
$$

Using the same procedure, for $k \geq 3$, we obtain :

$$
u^{3}=u^{4}=\ldots=u^{p}=\ldots=x \sin t
$$

Therefore, the exact solution of (55) is :

$$
u(x, t)=\lim _{k \rightarrow+\infty} u^{k}(x, t)=x \sin t
$$

\section{Conclusion}

In this work, we applied the SBA and Laplace-SBA methods to solve one mixed Volterra-Fredholm non-linear integro-differential equations and two other integrodifferential equations. The numerical results found show that these methods a re simple, more efficient and does not rely excessively on computer processing, as is the case with the Galerkin method and the collocations method. However, Laplace-SBA method applied to our integrodifferential equations gives exact solutions. The LaplaceSBA method therefore represents a particular interest by its rational and logical conception of a numerical model for solving integro-differential equations. Therefore, it can be used by researchers in engineering and physics for other equations or systems of Volterra differential equations. 


\section{References}

[1] Elbaz, E : Interactions fondamentales et structure de la matière. Herman, Paris (2002).

[2] Guy, B-L: Elements of mathematical physics. Addison Wesley, Reading (2013).

[3] Khajehnasiri A. A. Numerical Solution of Nonlinear 2D Volterra-Fredholm Integro-Differential Equations by Two-Dimensional Triangular Function. Int. Appl. Comput Math 2, 575-591 (2016).

[4] A. Tari, "On the Existence Uniqueness and Solution of the Nonlinear Volterra Partial Integro-Differential Equations", International Journal of Nonlinear Science, Vol. 16, No. 2, 152-163, (2013).

[5] Tari, A, Rahimi, M. Y, Shahmad, S., Talati, F. Development of the Tau method for the numerical solution of two -dimentional linear Voltera integrodifferential equations. J . Comput Method Appl-math. 9 (4), 421-435 (2009).

[6] Youssouf Pare1, Youssouf Minoungou, Abdoul Wassiha Nebie "Resolution of nonlinear convection - diffusion - reaction equations of Cauchy's kind by the Laplace SBA method",EUROPEAN JOURNAL OF PURE AND APPLIED MATHEMATICS Vol. 12, No. 3, 2019, 771789.

[7] Joseph Bonazebi Yindoula, Pare Youssouf, Gabriel Bissanga, Francis Bassono, Blaise Some. Application of the adomian decomposition method and the Some Blaise Abbo method to solving the diffusion-reaction equations. Advances in theorctical and applied mathematics, 9 (2): 97-104, 2014.

[8] G. Nkaya, F. Bassono, R. Yaro, J. Bonazebi Yindoula "Application of the SBA method for solving the partial differential equation" 10 (6) (2018) 98-107.

[9] O. Gonzalez J. Ruiz de Chavez and R. Bernal-Jaquez. Solution of the nonlinear kompaneets equation through the laplace-adomian decomposition method. Int. J.

[10] Appl. Comput. Math, 3 (2): 489-504, 2017.

[11] Bakodah HO. Anew modification of the LaplaceAdomian decomposition method for system of integral equations. J Am Sci. 2012; 8: 241-246.

[12] Wazwaz A. The combined Laplace transform-Adomian decomposition method for handling nonlinear Volterra integro-differential equations. Appl Math Comput. 2010; 216: 1304-1309.

[13] Darania, P., Shali, J., Ivaz, K.: New computational method for solving some 2-dimensional nonlinear Volterra integro-differential equations. Numer. Algorithm 57, 125-147 (2011).

[14] S. Ahmed and T. Elzaki. The solution of nonlinear volterra integro-differential equation of searel kind by combine Sumudu transforms and Adomian decomposition method. International Journal of Advanced and Inovative. Research 2 (2013) 90-93. 